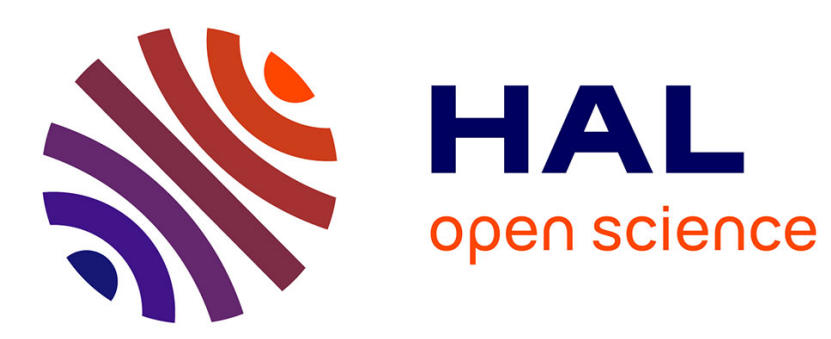

\title{
On necessary conditions of instability and design of destabilizing controls
}

\author{
Denis Efimov, Wilfrid Perruquetti, Mihály Petreczky
}

\section{To cite this version:}

Denis Efimov, Wilfrid Perruquetti, Mihály Petreczky. On necessary conditions of instability and design of destabilizing controls. IEEE CDC2014, Dec 2014, LA, United States. hal-01066282

\section{HAL Id: hal-01066282 \\ https://hal.inria.fr/hal-01066282}

Submitted on 19 Sep 2014

HAL is a multi-disciplinary open access archive for the deposit and dissemination of scientific research documents, whether they are published or not. The documents may come from teaching and research institutions in France or abroad, or from public or private research centers.
L'archive ouverte pluridisciplinaire HAL, est destinée au dépôt et à la diffusion de documents scientifiques de niveau recherche, publiés ou non, émanant des établissements d'enseignement et de recherche français ou étrangers, des laboratoires publics ou privés. 


\section{On necessary conditions of instability and design of destabilizing controls}

\begin{abstract}
The problem of formulation of an equivalent characterization for instability is considered. The necessary part of the Chetaev's theorem on instability is formulated. Using the developed necessary instability conditions, the Anti-control Lyapunov Function (ALF) framework from [1] is extended and the Control Chetaev Function (CCF) concept is proposed as a counterpart of the Control Lyapunov function (CLF) theory. A (bounded) control is designed, which destabilizes a nonlinear system based on CCF, this control design approach can be useful either for generation of an oscillating or chaotic behavior as in [1], or for analysis of norm controllability from [2].
\end{abstract}

\section{INTRODUCTION}

The problems of stability analysis and design of stabilizing controls for dynamical systems attracted a lot of attention during the last centenary [3], [4], [5], [6]. A rather rich theory has been developed, which is mainly based on the Lyapunov function method for stability analysis, and completed by the CLF approach for stabilization. It has been widely recognized that the Lyapunov function approach presents an equivalent (necessary and sufficient) formulation for these problems. Instability analysis, as counterpart of stability, has received also some attention, first by the works of Lyapunov [7], and next by the seminal contribution of Chetaev [8]. Some particular conditions of instability were obtained in [9] (see also [10]).

All previously mentioned results for instability were given as sufficient conditions only, the first attempt to show that for the case of a purely repulsing equilibrium the Lyapunov's conditions are also necessary, has been performed in [11], some necessary conditions for the instability results from [9] were derived in [12]. Next, the CLF approach has been extended in [1] for a destabilizing control design via the ALF framework. The utility of ALF and the design of destabilizing controls themselves was shown also in [1] on the example of control design for generation of oscillations in nonlinear systems. Another possible motivation for the interest in destabilizing controls is related with a recently introduced notion of the norm controllability [2], which is a property dual to input-to-state stability characterizing how far the state may go applying a bounded control.

Therefore, so far obtained necessary and sufficient conditions of instability deal with the Lyapunov case, where the equilibrium is purely repulsing (all trajectories in a vicinity of the equilibrium are escaping) and some special configurations from [9], where ad hoc restrictions are imposed on behavior of the trajectories into invariant cones around the equilibrium. All these cases are more restricted than the case studied by Chetaev [8] (the precise definition of this scenario of instability is given below). Thus the first goal of the present work is to develop the necessary conditions of the Chetaev's result, and next to extend the ALF framework appropriately.

The outline of this paper is as follows. The preliminaries are given in Section II. The necessary conditions of the Chetaev's theorem on instability are formulated in Section III. The extension of the ALF theory and universal formulas for destabilizing control design in bounded/unbounded setting are presented in Section IV. A summary and concluding remarks are in Section V.

\section{Preliminaries}

Define $\mathbb{R}_{+}=\{x \in \mathbb{R}: x \geq 0\}$, where $\mathbb{R}$ is the set of real numbers. The symbol $|s|$ is stated for the absolute value of a scalar $s \in \mathbb{R}$ or the Euclidean norm of a vector $s \in \mathbb{R}^{n}$. Denote $\mathcal{B}(\varepsilon)$ an open ball centered at the origin of the radius $\varepsilon \in \mathbb{R}_{+}$.

Let us consider a nonlinear dynamical system

$$
\dot{x}=f(x),
$$

where $x \in \mathbb{R}^{n}$ is the state vector, $f: \mathbb{R}^{n} \rightarrow \mathbb{R}^{n}$ is a locally Lipschitz continuous function, $f(0)=0$. A solution of this system for the initial condition $x_{0} \in \mathbb{R}^{n}$ at the time instant $t=0$ we will be denoted as $x\left(t, x_{0}\right)$ (i.e. $\left.x\left(0, x_{0}\right)=x_{0}\right)$, which is defined and unique for $t \in[0, T]$ with some $T \in$ $\mathbb{R}_{+}$. The system (1) is called forward complete if $T=+\infty$ for any $x_{0} \in \mathbb{R}^{n}$. A set $\mathcal{A} \subset \mathbb{R}^{n}$ is called invariant for (1) if $x\left( \pm t, x_{0}\right) \in \mathcal{A}$ for all $t \geq 0$ provided that $x_{0} \in \mathcal{A}$.

Let $\Omega$ be an open subset of $\mathbb{R}^{n}, 0 \in \Omega$.

Definition 1. [4] At the steady state $x=0$ the system (1) is said to be

(a) stable if for any $\epsilon>0$ there is $\delta(\epsilon)$ such that for any $x_{0} \in \Omega$, if $\left|x_{0}\right| \leq \delta(\epsilon)$ then $\left|x\left(t, x_{0}\right)\right| \leq \epsilon$ for all $t \geq 0$;

(b) asymptotically stable if it is stable and for any $\kappa>0$ and $\epsilon>0$ there exists $T(\kappa, \epsilon) \geq 0$ such that for any $x_{0} \in \Omega$, if $\left|x_{0}\right| \leq \kappa$ then $\left|x\left(t, x_{0}\right)\right| \leq \epsilon$ for all $t \geq T(\kappa, \epsilon)$;

(c) unstable if it is not stable, i.e. for any $\delta>0$ there exists $x_{0} \in \Omega=\mathcal{B}(\varepsilon)$ with $\left|x_{0}\right| \leq \delta$ such that $x\left(T_{x_{0}}, x_{0}\right) \notin \Omega$ for some $T_{x_{0}} \in \mathbb{R}_{+}$.

If $\Omega=\mathbb{R}^{n}$, then the corresponding properties are called global stability/asymptotic stability/instability of $x=0$ (in this case $T_{x_{0}} \in \mathbb{R}_{+} \cup\{+\infty\}$ ).

The notation $D V(x) f(x)$ stands for the directional derivative of a continuously differentiable function $V: \mathbb{R}^{n} \rightarrow \mathbb{R}_{+}$ with respect to the vector field $f$ evaluated at the point $x$, the upper and lower Dini derivatives in the direction of $f$ 
for a locally Lipschitz continuous function $V$ are defined as follows:

$$
\begin{aligned}
& D^{+} V(x) f(x)=\limsup _{h \rightarrow 0+} \frac{V(x+h f(x))-V(x)}{h}, \\
& D^{-} V(x) f(x)=\liminf _{h \rightarrow 0+} \frac{V(x+h f(x))-V(x)}{h} .
\end{aligned}
$$

The following theorem has been adapted from [8], [10].

Theorem 1. Let $V: \mathbb{R}^{n} \rightarrow \mathbb{R}$ be a locally Lipschitz continuous function, $V(0)=0$ and there exist $\epsilon_{0}>0$ such that $\mathcal{V}^{+} \cap \mathcal{B}(\epsilon) \neq \emptyset$ for any $\epsilon \in\left(0, \epsilon_{0}\right]$, where $\mathcal{V}^{+}=\left\{x \in \mathcal{B}\left(\epsilon_{0}\right): V(x)>0\right\}$. If $D^{-} V(x) f(x)>0$ for all $x \in \mathcal{V}^{+}$, then the origin is unstable for (1) with $\Omega=\mathcal{B}\left(\epsilon_{0}\right)$.

Note that in this theorem the Lipschitz continuity of $V(x)$ is needed on the set $\mathcal{V}^{+}$only, where the derivative $D^{-} V(x) f(x)$ is calculated.

\section{NECESSARY PART OF THE CHETAEV'S THEOREM}

Lemma 1. Consider a forward complete system (1) with a Lipschitz continuous function $f: \mathbb{R}^{n} \rightarrow \mathbb{R}^{n}, f(0)=0$ and denote by $\mathcal{A} \subset \mathbb{R}^{n}$ the invariant set for (1) (which contains all invariant solutions of the system). Then the following properties are equivalent for some $\epsilon_{0} \in \mathbb{R}_{+}$:

(i) The origin is unstable for (1) with $\Omega=\mathcal{B}\left(\epsilon_{0}\right)$ and $\mathcal{B}\left(\epsilon_{0}\right) \cap[\mathcal{A} \backslash\{0\}]=\emptyset$.

(ii) There is a continuous function $V: \mathcal{B}\left(\epsilon_{0}\right) \rightarrow \mathbb{R}_{+}$, $V(0)=0$, which is locally Lipschitz continuous on $\mathcal{V}^{+}=$ $\left\{x \in \mathcal{B}\left(\epsilon_{0}\right): V(x)>0\right\}$ and $\mathcal{V}^{+} \cap \mathcal{B}(\epsilon) \neq \emptyset$ for any $\epsilon \in$ $\left(0, \epsilon_{0}\right]$. In addition, $D^{-} V(x) f(x)>0$ for all $x \in \mathcal{V}^{+}$.

The proofs are omitted due to space limitation.

Remark 1. The same result can be obtained if we allow $V$ to be negative in the interior of the domain where $V(x)=0$. Applying standard smoothing arguments [13], existence of a smooth function $V$ can be proven in a similar way. Thus in the following consideration we will consider smooth Chetaev functions $V: \mathcal{B}\left(\epsilon_{0}\right) \rightarrow \mathbb{R}$ satisfying the conditions of Theorem 1 and Lemma 1, i.e. under these conditions existence of a smooth Chetaev function $V: \mathcal{B}\left(\epsilon_{0}\right) \rightarrow \mathbb{R}$ is necessary and sufficient for instability of (1) in $\mathcal{B}\left(\epsilon_{0}\right)$.

\section{DESTABILIZING CONTROLS}

Now consider a variant of system (1), affine in control input:

$$
\dot{x}=f(x)+G(x) u,
$$

where $x \in \mathbb{R}^{n}$ is the state vector, $u \in \mathbb{R}^{m}$ is the control, $f: \mathbb{R}^{n} \rightarrow \mathbb{R}^{n}$ and $G: \mathbb{R}^{n} \rightarrow \mathbb{R}^{m}$ are locally Lipschitz continuous functions, $f(0)=0$. Assume that a locally Lipschitz continuous control $u: \mathbb{R}^{n} \rightarrow \mathbb{R}^{m}$ is given, $u(0)=0$, such that the system (2) is unstable at the origin for $\Omega=\mathcal{B}\left(\epsilon_{0}\right)$ with some $\epsilon_{0}>0$ and $\mathcal{B}\left(\epsilon_{0}\right) \cap[\mathcal{A} \backslash\{0\}]=\emptyset$, where as before $\mathcal{A} \subset \mathbb{R}^{n}$ is the invariant set for (2) with $u=u(x)$ (by construction $0 \in \mathcal{A}$ ). Since all conditions of Lemma 1 are satisfied, then there is a smooth function
$V: \mathcal{B}\left(\epsilon_{0}\right) \rightarrow \mathbb{R}$, such that $V(0)=0$ and $\mathcal{V}^{+} \cap \mathcal{B}(\epsilon) \neq \emptyset$, $\mathcal{V}^{+}=\left\{x \in \mathcal{B}\left(\epsilon_{0}\right): V(x)>0\right\}$ for any $\epsilon \in\left(0, \epsilon_{0}\right]$, in addition for all $x \in \mathcal{V}^{+}$

$$
D^{-} V(x)[f(x)+G(x) u(x)]>0 .
$$

Such a Chetaev function $V$ is closely related with the ALF introduced in [1] for the case of globally repulsing equilibrium. As for the case of the autonomous system (1), a Lyapunov function can be used for a repulsing steady-state analysis (in this case $\dot{V}>0$ in a vicinity of the equilibrium) and a Chetaev function covers a more general case, when the repulsing trajectories belong to a part of the vicinity. In the present Chetaev's setting the definition of ALF can be extended as follows.

Definition 2. A smooth function $V: \mathcal{B}\left(\epsilon_{0}\right) \rightarrow \mathbb{R}$, such that $V(0)=0$ and $\mathcal{V}^{+} \cap \mathcal{B}(\epsilon) \neq \emptyset$ for any $\epsilon \in\left(0, \epsilon_{0}\right]$ where $\mathcal{V}^{+}=\left\{x \in \mathcal{B}\left(\epsilon_{0}\right): V(x)>0\right\}$, is called a Control Chetaev Function (CCF) for the system (2) if for all $x \in \mathcal{V}^{+}$,

$$
\sup _{u \in \mathbb{R}^{m}}\left\{a(x)+B(x)^{T} u\right\}>0,
$$

where

$$
a(x)=D^{-} V(x) f(x), B(x)=\left[D^{-} V(x) G(x)\right]^{T} .
$$

Such an CCF satisfies the Small Control Property (SCP) if for each $\epsilon>0$ there is a $\delta>0$ such that, if $x \in \mathcal{V}^{+}$satisfies $|x|<\delta$, then there is some $|u|<\epsilon$ such that

$$
a(x)+B(x)^{T} u>0 .
$$

Similarly to CLF case [14], it is possible to conclude that $V: \mathcal{B}\left(\epsilon_{0}\right) \rightarrow \mathbb{R}_{+}$is a CCF, if for all $x \in \mathcal{V}^{+}$the property

$$
|B(x)|=0 \quad \Longrightarrow \quad a(x)>0
$$

is satisfied. The SCP property for a CCF is equivalent to the limit condition [1]:

$$
\lim _{|x| \rightarrow 0} \frac{a(x)}{|B(x)|} \geq 0 .
$$

Obviously, if a CCF $V$ is positive definite (in this case $\mathcal{V}^{+}$ is an open set around the origin) and $\dot{V}>0$ everywhere in $\mathcal{V}^{+}$, then such a CCF becomes an ALF from [1] (that is an opposite of CLF from [14]), thus the CCF concept generalizes those previous results.

It easy to see that if $\epsilon_{0}<+\infty$ then any continuous control would be bounded on $\mathcal{B}\left(\epsilon_{0}\right)$ (very frequently, if excitation of oscillations is the goal, only local destabilization is needed [11], [1]), therefore as in [15] a CCF for bounded control inputs can be introduced. Denote $\mathbb{U}_{k}=\left\{u \in \mathbb{R}^{m}:|u| \leq k\right\}$ for some $k>0$.

Definition 3. A smooth function $V: \mathcal{B}\left(\epsilon_{0}\right) \rightarrow \mathbb{R}$, such that $V(0)=0$ and $\mathcal{V}^{+} \cap \mathcal{B}(\epsilon) \neq \emptyset$ for any $\epsilon \in\left(0, \epsilon_{0}\right]$ where $\mathcal{V}^{+}=\left\{x \in \mathcal{B}\left(\epsilon_{0}\right): V(x)>0\right\}$, is called a CCF for the system (2) with respect to controls from $\mathbb{U}_{k}$ if for all $x \in \mathcal{V}^{+}$,

$$
\sup _{u \in \mathbb{U}_{k}}\left\{a(x)+B(x)^{T} u\right\}>0,
$$


where

$$
a(x)=D^{-} V(x) f(x), B(x)=\left[D^{-} V(x) G(x)\right]^{T} .
$$

It is easy to prove that $V: \mathcal{B}\left(\epsilon_{0}\right) \rightarrow \mathbb{R}_{+}$is a CCF with respect to controls from $\mathbb{U}_{k}$, if for all $x \in \mathcal{V}^{+}$the properties

$$
\begin{gathered}
|B(x)|=0 \quad \Longrightarrow \quad a(x)>0, \\
a(x)>-k|B(x)|
\end{gathered}
$$

are satisfied.

In [14] a universal formula for stabilization using CLF has been proposed, in [1] it has been applied for destabilization with ALF. In this work below we are going to apply it for $\mathrm{CCF}$, and CCF with bounded controls using the universal formula given in [15].

Theorem 2. If for the system (2) there exists a CCF $U$ : $\mathcal{B}\left(\epsilon_{0}\right) \rightarrow \mathbb{R}$, then the control

$$
\begin{gathered}
u(x)=-\phi[a(x),|B(x)|] B(x), \\
\phi(a, b)= \begin{cases}\frac{a-\sqrt[p]{|a|^{p}+b^{2 q}}}{b^{2}} & \text { if } b \neq 0 ; \\
0 & \text { if } b=0\end{cases}
\end{gathered}
$$

for any $2 q \geq p>1, q>1$ is continuous for all $x \in \mathcal{V}^{+}$and ensures the system instability in $\mathcal{B}\left(\epsilon_{0}\right)$.

If furthermore $V$ satisfies the $S C P$, then the feedback control (3) is also continuous at the origin.

The control (3) can be designed to be homogeneous for a homogeneous system (2) under conditions similar to the ones formulated in [1].

Theorem 3. If for the system (2) there exists a CCF $V$ : $\mathcal{B}\left(\epsilon_{0}\right) \rightarrow \mathbb{R}$ with respect to controls from $\mathbb{U}_{k}$ for some $k \in$ $(0,+\infty)$, then the control

$$
\begin{gathered}
u(x)=-\varphi[a(x),|B(x)|] B(x), \\
\varphi(a, b)= \begin{cases}\frac{a-\sqrt[p]{|a|^{p}+b^{2 q}}}{b^{2}\left(1+\sqrt[p]{1+k^{-p} b^{2 q-p}}\right)} & \text { if } b \neq 0 ; \\
0 & \text { if } b=0\end{cases}
\end{gathered}
$$

for any $2 q \geq p>1, q>1$ is continuous for all $x \in \mathcal{V}^{+}$, $u(x) \in \mathbb{U}_{k}$ for $x \in \mathcal{V}^{+}$, and it ensures the system instability in $\mathcal{B}\left(\epsilon_{0}\right)$.

If furthermore $V$ satisfies the SCP, then the feedback control (4) is also continuous at the origin.

\section{CONCLUSION}

The necessary conditions of instability in terms of existence a Chetaev function are formulated. The obtained results are used for extension of the ALF framework from [1], and the CCF concept is introduced. With the proposed results, the CCF concept plays a similar role for feedback destabilization of dynamical systems as CLF has for stabilization. Two control algorithms are designed, based on the universal formulas of [15], [14], one is for design of a bounded locally destabilizing control. The developed approach can be applied next for characterization of the norm controllability [2].

\section{REFERENCES}

[1] D. Efimov and W. Perruquetti, "Oscillating system design applying universal formula for control," in Proc. 50th IEEE CDC-ECC 2011, (Orlando, FL), pp. 1747-1752, 2011.

[2] M. A. Müller, D. Liberzon, and F. Allgöwer, "Norm-controllability, or how a nonlinear system responds to large inputs," in Proc. 9th IFAC Symposium on Nonlinear Control Systems (NOLCOS), (Toulouse), pp. 104-109, 2013.

[3] A. Isidori, Nonlinear control systems: An Introduction. Berlin: Springer-Verlag, 2nd ed., 1989.

[4] H. Khalil, Nonlinear Systems. Upper Saddle River, New Jersey: Prentice Hall, 3rd ed., 2002.

[5] M. Krstić, I. Kanellakopoulos, and P. Kokotović, Nonlinear and Adaptive Control Design. Wiley \& Sons, Inc., 1995.

[6] R. Sepulchre, M. Jankovic, and P. Kokotović, Constructive nonlinear control. NY: Springer-Verlag, 1997.

[7] A. Lyapunov, The general problem of the stability of motion. London: Taylor \& Francis, 1992. Translated by A. T. Fuller.

[8] N. Chetaev, The Stability of Motion. New York: Pergamon Press, 1961. (English translation).

[9] L. Khazin and E. Shnol, Stability of Critical Equilibrium States. Manchester University Press, 1991.

[10] E. Shnol, "Chetaev function," Scholarpedia, vol. 2, no. 9, p. 4672 2007. www.scholarpedia.org/article/Chetaev $v_{\text {unction. }}$

[11] D. Efimov and A. Fradkov, "Oscillatority of nonlinear systems with static feedback," SIAM J. Control Optimization, vol. 48, no. 2, pp. 618640, 2009.

[12] D. Efimov and W. Perruquetti, "Oscillations conditions in homogenous systems," in Proc. IFAC NOLCOS Symp., (Bologna), pp. 1379-1384, 2010.

[13] Y. Lin, E. Sontag, and Y. Wang, "A smooth converse lyapunov theorem for robust stability," SIAM J. Control Optimization, vol. 34, pp. 124160,1996

[14] E. Sontag, "A "universal" construction of arstein's theorem on nonlinear stabilization," Systems \& Control Letters, vol. 12, pp. 542-550, 1989.

[15] Y. Lin and E. D. Sontag, "A universal formula for stabilization with bounded controls," Systems \& Control Letters, vol. 16, no. 6, pp. 393397, 1991. 